\title{
HARMONIC FORMS AND RIESZ TRANSFORMS FOR RANK ONE SYMMETRIC SPACES
}

\author{
BY AROLDO KAPLAN ${ }^{1}$ AND ROBERT PUTZ ${ }^{2}$
}

Communicated by Hans Weinberger, June 11, 1974

We study harmonic forms on a noncompact rank one symmetric space $M$; that is, differential forms satisfying the equations $d \omega=0, \delta \omega=0$. We define "Hardy spaces" $\mathbf{H}^{p}$ of harmonic forms on $M$ and study their boundary behavior. Fractional and singular integral operators are introduced on an Iwasawa group $\bar{N}$ of $M$, and used to characterize the boundary values of forms in $\mathbf{H}^{p}$, setting up an explicit isomorphism between these spaces and the ordinary $L^{p}$ spaces on $\bar{N}$. In this sense, these operators play a similar role to that of the Riesz transforms on Euclidean spaces and compact Lie groups associated to the "conjugate systems" of harmonic functions studied by Coifman, Stein, and Weiss [1], [7].

1. Some vector fields on $M$. Let $G$ be the identity connected component of the group of isometries of $M$; fix an Iwasawa decomposition $G=$ $K A N$ of $G$, and let $\bar{N}=\theta N$, where $\theta$ is the Cartan involution of $G$ associated to $K$. Let $\mathfrak{g}, \mathfrak{\imath}, \mathfrak{n}, \overline{\mathfrak{n}}, \mathfrak{a}$ be the Lie algebras of the groups $G, K, N$, $\bar{N}, A$. Now define a right-action $\tau$ of the solvable group $\bar{S}=\bar{N} A$ on $M=$ $G / K$ as follows: since $G=\bar{S} K$, each $x \in M$ can be written uniquely as $x=s \cdot o$, where $o=\{K\}, s \in \bar{S}$; then for $s^{\prime} \in \bar{S}$ let $\tau\left(s^{\prime}\right)(s \cdot o)=s s^{\prime} \cdot o$. For $X \in \overline{\mathbf{s}}$, considered as a left invariant vector field on $\bar{S}$, define a vector field $\widetilde{X}$ on $M$ by $\widetilde{X}_{\bar{n} a \cdot o}=\tau_{*}\left(\operatorname{Ad}\left(a^{-1}\right) X\right), \bar{n} \in \bar{N}, a \in A$, where $\tau_{*}$ denotes the infinitesimal action of $\bar{\delta}$ on $M$ induced by $\tau$. Since the action $\tau$ is free, $X \rightarrow \widetilde{X}$ maps a basis of $\bar{\delta}$ onto an everywhere defined frame of vector fields on $M$. Moreover, $[X, Y]^{\sim}=[\tilde{X}, \widetilde{Y}]$ whenever $X$ and $Y$ are both in $\overline{\mathfrak{n}}$ and $[\widetilde{X}, \widetilde{Y}]=0$ if $X \in \mathfrak{a}$. Note that the integral curves of $\widetilde{X}, X \in \mathfrak{a}$ are geodesics in $M$ which are orthogonal to the family of submanifolds $\bar{N} a \cdot o, a \in A$.

AMS (MOS) subject classifications (1970), Primary 43A85, 53C35; Secondary $35 \mathrm{H} 05$.

Key words and phrases. Harmonic forms, Lie groups, fundamental solution, singular and fractional integrals.

1 Partially supported by NSF grant GP 29704 A3.

2 Partially supported by NSF grant GP 28448.

Copyright $\odot 1975$, American Mathematical Society 
Let $\Delta_{+}$be the set of positive restricted roots of ad $a$ associated to the Iwasawa decomposition chosen above. Then $\overline{\mathfrak{n}}=\Sigma_{\alpha \in \Delta}+\overline{\boldsymbol{g}}_{\alpha}$, where $\overline{\mathrm{g}}_{\alpha}$ is the root space corresponding to the root $-\alpha$, and the decomposition $\bar{\delta}=$ $a+\Sigma_{\alpha \in \Delta_{+}} \bar{g}_{\alpha}$ is orthogonal with respect to the inner product $(X, Y)=$ $-B(X, \theta Y)$, with $B$ denoting the Killing form of $g$. Set $\rho=1 / 2 \Sigma_{\alpha \in \Delta_{+}} m_{\alpha} \alpha$, where $m_{\alpha}$ is the multiplicity of the root $\alpha$.

Note, in general, $\delta \omega=0=d \omega$ does not imply that the functions $\omega(\widetilde{X}), X \in \bar{\delta}$, are annihilated by the Laplace-Beltrami operator $\Delta$. The following result, however, provides an opening for the application of Fatou type theorems to the present context:

Proposition 1. If $d \omega=0=\delta \omega$ and $X$ belongs to the center of $\overline{\mathfrak{n}}$, then $\Delta \omega(\widetilde{X})=0$.

2. Riesz transforms on $\bar{N}$. Henceforth we assume that $\operatorname{rank} M=1$. We can then choose a root $\alpha \in \Delta_{+}$such that either $\Delta_{+}=\{\alpha\}$ or $\Delta_{+}=$ $\{\alpha, 2 \alpha\}$, so that $\overline{\mathfrak{n}}=\overline{\mathfrak{g}}_{\alpha}+\overline{\mathfrak{g}}_{2 \alpha}$. Set $m=m_{\alpha}+2 m_{2 \alpha}$; in order to avoid technical complications it will be convenient to assume that $m \geqslant 3$ (see the remark at the end).

If $X \in \mathfrak{g}$, let $|X|=(X, X)^{1 / 2}$. Now, for $\epsilon \geqslant 0$, define the function

$$
\langle\bar{n}\rangle_{\epsilon}=\left(\eta|X|^{2}+\epsilon^{2}\right)^{2}+|Y|^{2},
$$

where $\bar{n} \in \bar{N}, \bar{n}=\exp (X+Y), X \in \overline{\mathfrak{g}}_{\alpha}, Y \in \overline{\mathrm{g}}_{2 \alpha} ; \eta$ denotes the constant $(\alpha, \alpha) / 8=\left(16\left(m_{\alpha}+4 m_{2 \alpha}\right)\right)^{-1}$.

Consider now the differential operator $D=\Sigma_{i} X_{i}^{2}$ on $\bar{N}$, where $X_{1}, X_{2}, \cdots, X_{m_{\alpha}}$ is an orthonormal basis of $\bar{g}_{\alpha}$; this operator is clearly independent of the choice of the basis $\left\{X_{i}\right\}$.

Proposition 2. $D\langle\bar{n}\rangle_{\epsilon}^{-(m-2) / 4}=-(m-2) m_{\alpha} \eta \epsilon^{2}\langle\bar{n}\rangle_{\epsilon}^{-(m+2) / 4}$.

The proof is a straightforward computation using the following

LEMMA (arbitrary rank). Let $\beta$ be a restricted root and $\left\{X_{i}\right\}$ an orthonormal basis of $\mathfrak{g}_{\beta}$. Then, for $X \in \mathfrak{g}_{\beta}, Y \in \mathfrak{g}_{2 \beta}$

(i) $\Sigma_{i}\left|\left[X, X_{i}\right]\right|^{2}=2 m_{2 \beta}(\beta, \beta)|X|^{2}$;

(ii) $\Sigma_{i}\left(Y,\left[X, X_{i}\right]\right)^{2}=2(\beta, \beta)|X|^{2}|Y|^{2}$.

Now put

$$
c^{-1}=-m_{\alpha}(m-2) \eta \cdot \int_{\bar{N}}\langle\bar{n}\rangle_{1}^{-(m+2) / 4} d \bar{n}
$$


and define the function

$$
G(\bar{n})=c\langle\bar{n}\rangle_{0}^{-(m-2) / 4}
$$

on $\bar{N}$.

Proposition 3. $G(\bar{n})$ is a fundamental solution for $D$.

RemarKs. (1) The operator $D$ is not elliptic except, of course, when $\overline{\mathrm{g}}_{2 \alpha}=(0)$. Proposition 3 shows, however, that $D$ is always hypoelliptic. This is also a consequence of the fact that the root space $\overline{\boldsymbol{g}}_{\alpha}$ generates the Lie algebra $\overline{\mathfrak{n}}$ (see [3]).

(2) For the Heisenberg group this fundamental solution was obtained by Folland [2].

Now, for each element $Z \in \overline{\mathfrak{n}}$, define the associated "Riesz kernel" $r_{Z}$ and the "Riesz transform" $R_{Z}$ on $\bar{N}$ by

$$
r_{Z}(\bar{n})=Z G(\bar{n}), \quad R_{Z} f(\bar{n})=f * r_{Z}(\bar{n})=\int_{\bar{N}} f(g) r_{Z}\left(g^{-1} \bar{n}\right) d g .
$$

A straightforward computation gives, for $Z \in \overline{\boldsymbol{g}}_{\alpha}$,

$$
r_{Z}(\bar{n})=1 / 2(2-m) c\langle\bar{n}\rangle_{0}^{-(m+2) / 4} \cdot\left(2 \dot{\eta}|X|^{2}(X, Z)+1 / 2(Y,[X, Z])\right),
$$

and for $Z \in \overline{\mathbf{g}}_{2 \alpha}$,

$$
r_{Z}(\bar{n})=1 / 2(2-m) c\langle\bar{n}\rangle_{0}^{-(m+2) / 4}(Y, Z)
$$

where $\bar{n}=\exp (X+Y), X \in \overline{\mathrm{g}}_{\alpha}, Y \in \overline{\mathrm{g}}_{2 \alpha}$.

THEOREM 1. The Riesz transform $R_{Z}$ is

(i) a bounded operator from $L^{p}(\bar{N})$ into $L^{p}(\bar{N}), 1<p<\infty$, if $Z \in \overline{\mathrm{g}}_{2 \alpha}$, and

(ii) a bounded operator from $L^{p}(\bar{N})$ into $L^{q}(\bar{N}), 1<p<q<\infty$, $q^{-1}=p^{-1}-m^{-1}$, if $Z \in \overline{\mathrm{g}}_{\alpha}$.

For the proof, observe that the functions $r_{Z}$ are singular integral kernels (if $Z \in \overline{\mathfrak{g}}_{2 \alpha}$ ) and fractional integral kernels (if $Z \in \overline{\mathfrak{g}}_{\alpha}$ ). They satisfy the following homogeneity properties under the "dilations" of $\bar{N}$ induced by $A$ :

$$
\begin{array}{ll}
r_{Z}\left(a^{-1} n a\right)=e^{-(m-1) \alpha(\log a)} \cdot r_{Z}(\bar{n}) & \left(Z \in \bar{g}_{\alpha}\right), \\
r_{Z}\left(a^{-1} n a\right)=e^{-m \alpha(\log a)} \cdot r_{Z}(\bar{n}) & \left(Z \in \bar{g}_{2 \alpha}\right),
\end{array}
$$

and are odd for $Z$ in $\bar{g}_{2 \alpha}$. 
General theorems about these type of operators on nilpotent groups have also been obtained by Korányi-Vági [5] and Stein [6].

Observe that the Riesz transforms satisfy the following relations:

$$
\begin{aligned}
X R_{Y}-Y R_{X}=R_{[X, Y]} & (X, Y \in \overline{\mathfrak{n}}), \\
\sum_{i} X_{i} \circ R_{X_{i}}=\text { identity } & \left(\left\{X_{i}\right\} \text { an orthonormal basis of } \overline{\mathbb{g}}_{\alpha}\right) .
\end{aligned}
$$

3. Boundary values of harmonic forms. Let $H$ be the unique element in a such that $\alpha(H)=1$, and define the vector field $\widetilde{W}$ on $M$ by

$$
\widetilde{W}_{\bar{n} a \cdot o}=e^{2 \alpha(\log a)} \widetilde{H}_{n a \cdot o} \quad(n \in \bar{N}, a \in A) .
$$

For each $p, 1<p<m$, let $\mathbf{H}^{p}$ be the space of all 1 -forms $\omega$ on $M$ such that:

(a) $d \omega=0=\delta \omega$,

(b) $\omega(\widetilde{W}), \omega(\widetilde{Y}) \in L^{p} \quad\left(Y \in \overline{\mathfrak{g}}_{2 \alpha}\right)$,

(c) $\omega(\widetilde{X}) \in L^{q} \quad\left(X \in \bar{g}_{\alpha}, q^{-1}=p^{-1}-m^{-1}\right)$.

With $\bar{N}$ regarded as a boundary for the symmetric space $M$ (see e.g. [4]), we can now state the following results.

THEOREM 2. Let $f \in L^{p}(\bar{N}), 1<p<m$, and define a 1-form $\omega_{f}$ on $M$ by letting $\omega_{f}(\widetilde{W})=f * \widetilde{W}(P * G), \omega_{f}(\widetilde{X})=f * P * r_{X}, X \in \overline{\mathfrak{n}} . \quad(P$ is the Poisson kernel, $G$ is the fundamental solution of $D, r_{X}$ the kernel constructed above.) Then

(i) $\omega_{f} \in \mathbf{H}^{p}$.

(ii) $\omega_{f}(\widetilde{W})$ converges in $L^{p}$ and almost everywhere to $f$.

(iii) $\omega_{f}(\widetilde{X})$ converges in $L^{q}$ (respectively $L^{p}$ ) and almost everywhere to $f * r_{X}$ if $X \in \overline{\mathbf{g}}_{\alpha}$ (respectively $X \in \overline{\mathbf{g}}_{2 \alpha}$ ).

THEOREM 3. If $\omega \in \mathbf{H}^{p}$, then there exists a (unique) $f \in L^{p}(\bar{N})$ such that $\omega=\omega_{f}$.

REMARK. In order to obtain analogous results when $m<3$ (real hyperbolic spaces of dimension 2 or 3), one must modify the definition of the corresponding $\mathbf{H}^{p}$ spaces and Riesz transforms; in dimension 2 this leads to the classical connection between conjugate harmonic functions in the upper half-plane and the Hilbert transform in the line.

A detailed version of these results will appear elsewhere. 


\section{REFERENCES}

1. R. R. Coifman and G. Weiss, Invariant systems of conjugate harmonic functions associated with compact Lie groups, Studia Math. 44 (1972).

2. G. B. Folland, A fundamental solution for a subelliptic operator, Bull. Amer. Math. Soc. 79 (1973), 373-376. MR 47 \#3816.

3. L. Hörmander, Hypoelliptic second order differential equations, Acta Math. 119 (1967), 147-171. MR 36 \#5526.

4. A. Korányi, Boundary behavior of Poisson integrals on symmetric spaces, Trans. Amer. Math. Soc. 140 (1969), 393-409. MR 39 \#7132.

5. A. Korányi and S. Vági, Singular integrals on homogeneous spaces and some problems of classical analysis, Ann. Scuola Norm. Sup. Pisa 25 (1971).

6. E. M. Stein, Singular integrals and estimates for the Cauchy-Riemann equations, Bull. Amer. Math. Soc. 79 (1973), 440-445. MR 47 \#3851.

7. E. M. Stein and G. Weiss, On the theory of harmonic functions of several variables. I. The theory of HP-spaces, Acta Math. 103 (1960), 25-62. MR 22 \#12315.

DEPARTMENT OF MATHEMATICS, COLUMBIA UNIVERSITY, NEW YORK, NEW YORK 10027

DEPARTMENT OF MATHEMATICS, BELFER GRADUATE SCHOOL OF SCIENCE, YESHIVA UNIVERSITY, NEW YORK, NEW YORK 10033

Current address (Aroldo Kaplan): Department of Mathematics, University of Massachusetts, Amherst, Massachusetts 01002 